\title{
A Geometrical Approach for the Optimal Control of Sequencing Batch Bio-Reactors
}

\author{
N. Abdellatif ${ }^{1,2, *}$, W. Bouhafs ${ }^{2,3}$, J. Harmand ${ }^{4}$, F. Jean ${ }^{5}$ \\ ${ }^{1}$ Manouba University, ENSI, Campus Universitaire de Manouba, 2010 Manouba, Tunisia \\ 2 Tunis El Manar University, ENIT, LAMSIN, BP 37, Le Belvédère, 1002 Tunis, Tunisia \\ ${ }^{3}$ Jendouba University, FSJEGJ, Avenue de l'U.M.A, 8189 Jendouba, Tunisia \\ ${ }^{4}$ Montpellier University, INRAE, LBE, Narbonne, France \\ ${ }^{5}$ Paris-Saclay University, UMA, ENSTA ParisTech, 828 bd des Maréchaux, 91762 Palaiseau, France
}

\begin{abstract}
In this work, we consider an optimal control problem of a biological sequencing batch reactor (SBR) for the treatment of pollutants in wastewater. This model includes two biological reactions, one being aerobic while the other is anoxic. The objective is to find an optimal oxygen-injecting strategy to reach, in minimal time and in a minimal time/energy compromise, a target where the pollutants concentrations must fulfill normative constraints. Using a geometrical approach, we solve a more general optimal control problem and thanks to Pontryagin's Maximum Principle, we explicitly give the complete optimal strategy.
\end{abstract}

Keywords Sequencing batch reactor (SBR), Optimal control, Minimal time problem, Pontryagin's Maximum Principle, Optimal synthesis.

DOI: $10.19139 /$ soic-2310-5070-868

\section{Introduction}

The biological treatment of organic and/or chemical pollutants contained in wastewaters is the transformation of the biodegradable material (also called biomass) in sludge. The principle of biological treatment is to put together microorganisms and pollutants in reactors in which it is possible to control environmental conditions. Due to the simplicity of its implementation, the biological pathway for the treatment of wastewaters is widely used. In this process, bacteria agglomerate into flocs in the reactive part of the system while in a second part, in the absence of agitation, they settle under the effect of gravity. The sludge is thus separated from the treated water and recycled or withdrawn, while the clean water is rejected in the environment.

Several technologies using these principles for the biological treatment of wastewaters have been developed. One such technology relies on the biological Sequencing Batch Reactors (SBR). By separating in time rather than in space the above-mentioned treatment and separation phases, the SBRs, compared to their continuous counterparts, allow a better control of the process and therefore a better quality of the treated effluent.

The price to pay is that the considered system is operating in batch rather than in continuous mode. Therefore, it requires the use of an upstream storage basin for wastewaters which arrive continuously to the treatment plant.

As a consequence, a major issue linked to this process is to minimize the time during which the process is unavailable, i.e. the total reaction time required to treat a batch of wastewater, under the assumption that the settling time is constant. The biological treatment of waters requires different aeration conditions in order to remove both organic carbon and nitrogen compounds. Several optimal control algorithms for the optimization of SBRs have

\footnotetext{
${ }^{*}$ Correspondence to: N. Abdellatif (Email:nahla.abdellatif@ensi-uma.tn). Manouba University, ENSI, Campus Universitaire de Manouba,
} 2010 Manouba, Tunisia.

ISSN 2310-5070 (online) ISSN 2311-004X (print)

Copyright (C) 2021 International Academic Press 
been proposed, [7, 9]. Classical tools of optimal control theory (PMP, direct methods, shooting methods, ...) allowed also to provide control strategies for SBRs. These tools are also utilized for optimal control in many real chemical, biological and physical processes as in [1] and [6]. In [8], a strategy to determine the switching instants between aerobic and anoxic phases is available.

Since the manipulated variable is the aeration mode of operation (presence or absence of oxygen), such a process can be described by continuous-time dynamical systems and a control (here the oxygen concentration) that affects the system dynamics. In [7], only purely aerobic (saturation of oxygen concentration) or purely anoxic (no oxygen injection) conditions were modeled. However, oxygen regulation systems are now available.

Therefore, we propose in this paper to consider an optimal control problem in minimal time where the oxygen concentration may be kept low enough to allow both aerobic and anoxic reactions to occur simultaneously. This simultaneity of the reactions is classic in activated sludge processes and well modeled in ASM models of the IWA, see [5]. It is worth to investigate if such oxygen modulation approach could be better than the alternation of purely aerobic/anoxic phases as proposed in [8]. Indeed, in such a process which can be viewed as a switching system because of the switching aeration mode of operation (presence or absence of oxygen), the major difficulty relies on the existence of an optimal control. Most of the solutions for this type of systems consist in reformulating the problem and augmenting it into a larger family of systems. Besides, the optimal solution, when it exists, is a concatenation of a limited number of a "bang-singular-bang" controls, in several situations. When the singular control is not allowed, it is approximated by successive switches which cannot be applied for many systems because of some physical limitation or model robustness limitation at high switching frequency. That's why, we rather propose to consider a system allowing both aerobic and anoxic reactions to occur simultaneously.

This problem requires considering a three-dimensional model for which the control problem is very difficult to solve directly. That is why we rather propose to study first a slightly different problem in which the substrates are not explicitly coupled as in [7]. In particular, we are interested here in a model in which the oxygen concentration is the control variable and where, as in the three-dimensional case, both biological reactions - which can occur simultaneously - are necessary to treat two different types of substrates. The problem in two dimensions is expressed mathematically by an optimal control problem where the dynamics is a two-dimensional differential system with decoupled state variables. This system models the variations of concentrations of two pollutants. Our objective is to find an optimal control strategy to reach a target set, by minimizing a cost function that is expressed as an integral of a positive function. This problem has been addressed in $[3,4]$ with quite specific assumptions on the growth functions (convexity, values on the edges of the target, etc ...). However, these assumptions rendered the model quite unrealistic. For this reason, we propose to relax the conditions on the dynamic system and to consider more general biological reactions.

The technique we use in this work consists in formulating the optimal control problem as a geometric optimization problem in the plane. Solving this geometric problem allows us to weaken the assumptions on the growth functions and on the cost function and to completely solve the considered problems.

The paper is organized as follows. Section 2 is devoted to the statement of a class of optimal control problem. In Section 3, we solve these problems through a complete analysis of the convexified problem. In Section 4, we consider a model where two chemical or biological harmful substances (substrates) are consumed by two microorganisms (biomasses). Both microbial biomasses are limited and inhibited by their respective substrate, one being promoted by oxygen (aerobic reaction) while the second is inhibited by oxygen (anoxic reaction). We describe the control strategy and analyse the dependance with respect to the compromise time/energy in the cost function.

\section{Two-dimensional optimal control problems}

The reduced two-dimensional dynamics we consider are control systems of the form

$$
\left\{\begin{array}{l}
\dot{s}_{1}=-f_{1}(u) \rho_{1}\left(s_{1}\right), \\
\dot{s}_{2}=-f_{2}(u) \rho_{2}\left(s_{2}\right),
\end{array}\right.
$$


where the state $s=\left(s_{1}, s_{2}\right)$ belongs to $(0,+\infty)^{2}$ and the control variable $u$ belongs to an interval $\left[0, u_{\max }\right]$ with $u_{\max }>0$. The admissible controls are the $L^{\infty}$ functions $u$ defined on an interval $[0, t(u)]$ with values in $\left[0, u_{\max }\right]$, we write for short $u(\cdot) \in L^{\infty}\left([0, t(u)],\left[0, u_{\max }\right]\right)$. The functions $\rho_{i}$ and $f_{i}, i=1,2$, are assumed to satisfy the following hypothesis.

\section{Hypothesis $\mathbf{H}$}

- $\rho_{1}$ and $\rho_{2}$ are smooth on an open interval containing $[0,+\infty)$, are positive on $(0,+\infty)$, and are zero at 0 , $\rho_{1}(0)=\rho_{2}(0)=0$

- $f_{1}, f_{2}$ are nonnegative continuous functions on an open interval containing $\left[0, u_{\max }\right]$.

Clearly, this hypothesis guarantees existence and unicity of the solutions of (1) for fixed initial conditions and control function, as well as the invariance of the positive quadrant $(0,+\infty)^{2}$. Note also that the variables $s_{i}$ are always non increasing.

We consider a square $\operatorname{target} \mathcal{C}_{0}$ defined by:

$$
\mathcal{C}_{0}=\left[0, a_{1}\right] \times\left[0, a_{2}\right],
$$

where $a_{1}$ and $a_{2}$ are positive numbers, and a continuous nonnegative function $J$ defined on $\left[0, u_{\max }\right]$. Given an initial condition $s^{0} \in(0,+\infty)^{2}$, we introduce the following optimal control problem in free time,

$$
\left(\mathcal{P}_{0}\right)\left\{\begin{array}{l}
\min C(u)=\int_{0}^{t(u)}(1+J(u(\tau))) d \tau \\
u(\cdot) \in L^{\infty}\left([0, t(u)],\left[0, u_{\max }\right]\right), \\
s(\cdot) \text { solution of }(1) \text { s.t. } \quad s(0)=s^{0} \text { and } s(t(u)) \in \mathcal{C}_{0} .
\end{array}\right.
$$

When $J \equiv 0$, this is a minimum time problem. However even when $J \not \equiv 0$, it is possible to consider $\left(\mathcal{P}_{0}\right)$ as a minimum time problem up to a modification of the control system. And actually we can simplify drastically the problem thanks to change of variables and of time that we describe now.

Consider the change of variables $s \mapsto S$ defined by:

$$
S_{1}(t)=\int_{s_{1}(t)}^{s_{1}^{0}} \frac{d s}{\rho_{1}(s)} \quad \text { and } \quad S_{2}(t)=\int_{s_{2}(t)}^{s_{2}^{0}} \frac{d s}{\rho_{2}(s)} .
$$

In coordinates $S$ the target writes as (see Figure 1)

$$
\mathcal{C}_{\bar{S}}=\left\{\left(S_{1}, S_{2}\right) \in \mathbb{R}^{2} \text { s.t. } S_{1} \geq \bar{S}_{1} \text { and } S_{2} \geq \bar{S}_{2}\right\}, \quad \text { where } \bar{S}_{1}=\int_{a_{1}}^{s_{1}^{0}} \frac{d s}{\rho_{1}(s)}, \bar{S}_{2}=\int_{a_{2}}^{s_{2}^{0}} \frac{d s}{\rho_{2}(s)} .
$$

Now, for a given $u(\cdot)$, set:

$$
\tau(t)=\int_{0}^{t}(1+J(u(s))) d s, \quad t \in[0, t(u)]
$$

Using $\tau$ as time-parameter, the function $S(\tau)$ is solution of the control system

$$
\dot{S}=F(u), \quad \text { where } \quad F(u)=\left(\frac{f_{1}(u)}{1+J(u)}, \frac{f_{2}(u)}{1+J(u)}\right) .
$$

Noticing that $\tau(t(u))=C(u)$, the optimal control problem $\left(\mathcal{P}_{0}\right)$ can be written as:

$$
(\mathcal{P})\left\{\begin{array}{l}
\min \tau(u), \\
u(\cdot) \in L^{\infty}\left([0, \tau(u)],\left[0, u_{\max }\right]\right), \\
S(\cdot) \text { solution of }(3) \text { s.t. } \quad S(0)=0 \quad \text { and } S(\tau(u)) \in \mathcal{C}_{\bar{S}} .
\end{array}\right.
$$




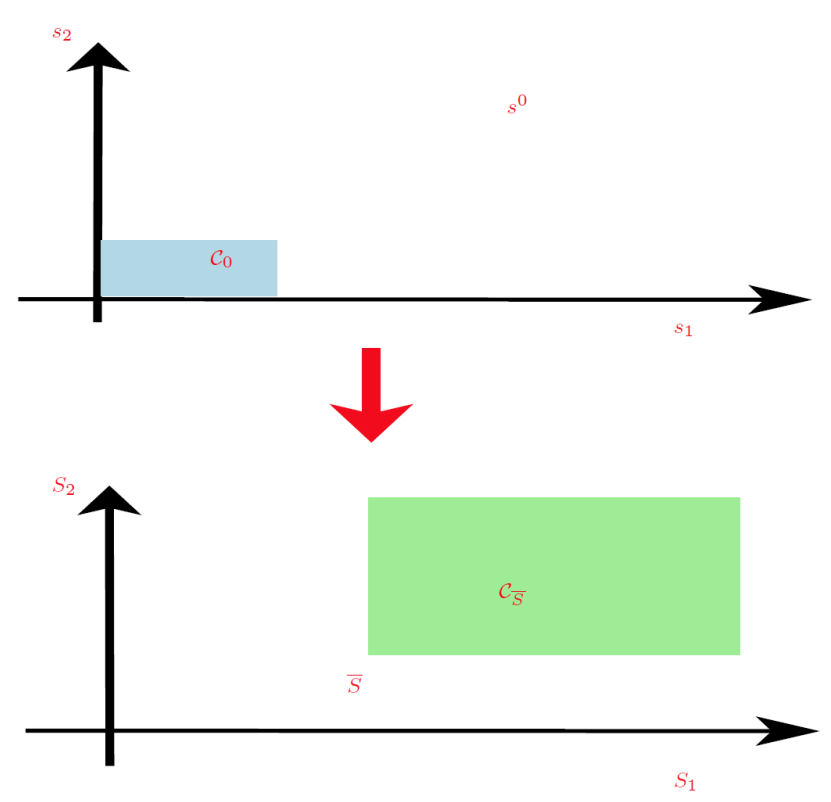

Figure 1. Transition from $\mathcal{C}_{0}$ to $\mathcal{C}_{\bar{S}}$

Note that the problems $\left(\mathcal{P}_{0}\right)$ and $(\mathcal{P})$ are equivalent in the sense that the minimum value of their objective functions are equal, and that the optimal solutions $u(\cdot)$ (if they exists) are the same for both problems up to timereparameterization. It is then sufficient to solve the simplified problem $(\mathcal{P})$, which will be the object of the next section. Before doing so, let us make two remarks.

- If $s_{i}^{0} \leq a_{i}(i=1$ or 2$)$, then $\bar{S}_{i} \leq 0$. In this case the problem is trivial (one-dimensional problem). Moreover, in the applications the target is considered to be small w.r.t. the initial values of the state. Thus in the sequel we always assume $\bar{S}_{i}>0$ for $i=1,2$, that is, $\bar{S} \in(0,+\infty)^{2}$.

- The problem $(\mathcal{P})$ is not convex in general. As a consequence, the existence of optimal solutions is not guaranteed by the usual theorems.

The second remark suggests to solve first the problem for the convexified system,

$$
\dot{S} \in \operatorname{conv}\left\{F(u): u \in\left[0, u_{\max }\right]\right\},
$$

where $\operatorname{conv}(A)$ denotes the convex hull of the set $A$.

\section{Resolution by convexification}

In this section we first solve a class of convex minimum time problems (subsections 3.1 to 3.3), and we use these results in 3.4 to solve Problems $(\mathcal{P})$ and $\left(\mathcal{P}_{0}\right)$.

\subsection{The convex problem $\left(\mathcal{P}_{c o}\right)$}

Let $V$ be a compact and convex subset of $\mathbb{R}_{+}^{2}$ having a non-empty intersection with the positive quadrant, i.e. $V \cap(0,+\infty)^{2} \neq \emptyset$. We introduce the control system defined by:

$$
\dot{S}=v, \quad v \in V
$$


and the associated minimum time problem,

$$
\left(\mathcal{P}_{c o}\right)\left\{\begin{array}{l}
\min t(v), \\
v(\cdot) \in L^{\infty}([0, t(v)], V), \\
S(\cdot) \text { solution of }(4) \text { s.t. } S(0)=0 \text { and } S(t(v)) \in \mathcal{C}_{\bar{S}} .
\end{array}\right.
$$

Note that for the control system (4), the set of states that are reachable from 0 is the cone $\mathbb{R}_{+} V$. Since we have assumed $\bar{S} \in(0,+\infty)^{2}$ and $V \cap(0,+\infty)^{2} \neq \emptyset$, this reachable set intersects the target $\mathcal{C}_{\bar{S}}$. It then results from standard results (for instance [10, Th. 6.2.1]) that there exists optimal solutions to the problem $\left(\mathcal{P}_{c o}\right)$.

Let us now apply Pontryagin's Maximum Principle (see [11] for instance). We introduce first the Hamiltonian $H:=H\left(S, p, p^{0}, v\right)$ where $p=\left(p_{1}, p_{2}\right) \in \mathbb{R}^{2}$ and $p^{0} \in \mathbb{R}$, as

$$
H=p^{T} v-p^{0}=p_{1} v_{1}+p_{2} v_{2}-p^{0} .
$$

Consider now an optimal control $v(\cdot)$ and the associated trajectory $S(\cdot)$. There exist $t(v):=t_{f}>0, p^{0} \geq 0$, and $p:\left[0, t_{f}\right] \rightarrow \mathbb{R}^{2}$ satisfying $\left(p(\cdot), p^{0}\right) \neq(0,0)$ and

$$
\dot{p}=-\frac{\partial H}{\partial S}, \quad \text { that is, } \quad \dot{p}=0 .
$$

Moreover, the following maximization condition holds:

$$
H\left(S(t), p, p^{0}, v(t)\right)=\max _{w \in V} H\left(S(t), p, p^{0}, w\right)=0 \quad \text { for a.e. } t \in\left[0, t_{f}\right] .
$$

The two conditions above imply

$$
p_{1} v_{1}(t)+p_{2} v_{2}(t)-p^{0}=0, \quad \forall t \in\left[0, t_{f}\right] .
$$

Besides, the following transversality condition is satisfied:

$$
\left\langle p, z-S\left(t_{f}\right)\right\rangle \geq 0, \quad \forall z \in \mathcal{C}_{\bar{S}}
$$

which implies that $p \in\left(\mathbb{R}_{+}\right)^{2}$.

To summarize, with every optimal trajectory $S(\cdot)$ is associated a four-tuple

$$
\left(S(\cdot), p, p^{0}, v(\cdot)\right)
$$

which satisfies all preceding conditions. Such a four-tuple is called an extremal, and its first component $S(\cdot)$ an extremal trajectory. An extremal is said to be normal if $p^{0} \neq 0$, abnormal otherwise.

\subsection{Analysis of the extremals of $\left(\mathcal{P}_{c o}\right)$}

\section{Lemma 1}

There are no abnormal extremals for problem $\left(\mathcal{P}_{c o}\right)$.

Proof

Assume that $p^{0}=0$. Then $p \neq 0$, and from (7), we have $p^{T} v(\cdot) \equiv 0$. It follows that the adjoint vector $p \in\left(\mathbb{R}_{+}\right)^{2}$ is orthogonal to $v$, which implies that $v(t)$ is either horizontal or vertical for all $t \in\left[0, t_{f}\right]$. In both cases, the extremal trajectory starting at the point $S(0)=0$ stays on one of the axis $\left\{S_{1}=0\right\}$ or $\left\{S_{2}=0\right\}$, and so can not reach the target.

Hence $p^{0}>0$ and, by (7), $p \neq 0$. We can multiply the Hamiltonian $H$ by $1 /\|p\|$ and assume that $p$ belongs to the quarter of the unit circle $S^{1+}=S^{1} \cap\left(\mathbb{R}_{+}\right)^{2}$. 


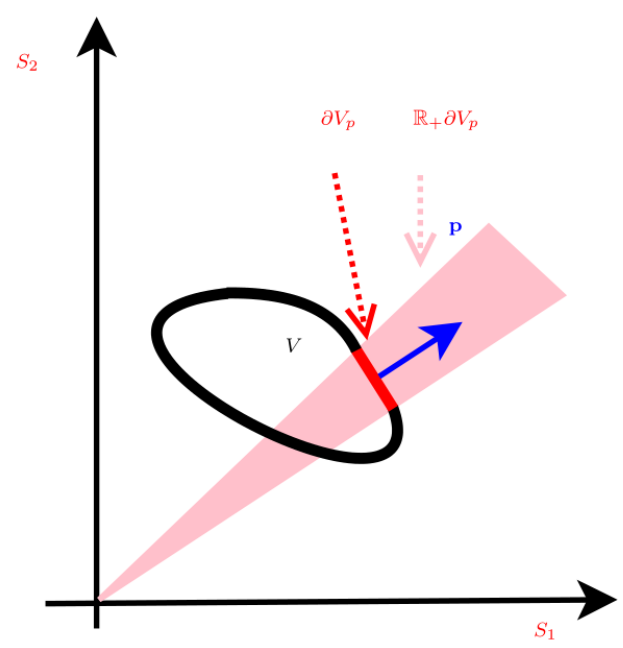

Figure 2. The sets $V, \partial V_{p}$ and $\mathbb{R}_{+} \partial V_{p}$

The condition (6) on $p$ can be written as

$$
v(t) \in \partial V_{p}=\underset{w \in V}{\operatorname{argmax}} p^{T} w, \quad \text { for } \quad \text { a.e. } \quad t \in\left[0, t_{f}\right] .
$$

Geometrically, $\partial V_{p}$ is the subset of points in $V$ that have the largest coordinate $p$ in the base $\left(p, p^{\perp}\right)$. In other words, $\partial V_{p}$ is the part of the boundary of $V$ where the outward normal vector is $p$, see Figure 2. Since $V$ is convex, $\partial V_{p}$ is either a singleton or a line segment. Note also that, when $p$ is one of the vectors $e_{1}, e_{2}$ of the canonical basis, then $\partial V_{e_{1}}=\left\{v \in V: v_{1}=v_{1}^{\max }\right\}$ and $\partial V_{e_{2}}=\left\{v \in V: v_{2}=v_{2}^{\max }\right\}$, where $v_{1}^{\max }=\max \left\{v_{1}:\left(v_{1}, v_{2}\right) \in V\right\}$ and $v_{2}^{\max }=\max \left\{v_{2}:\left(v_{1}, v_{2}\right) \in V\right\}$.

\section{Remark 1}

We could also define $\partial V_{p}$ in terms of supporting plane. Recall that the support function of $V$ is the function $h_{V}: \mathbb{R}^{2} \rightarrow \mathbb{R}$ given by

$$
h_{V}(p)=\sup \left\{p^{T} v: v \in V\right\},
$$

and that, for $p \in S^{1}$, the set $H(p)=\left\{v \in \mathbb{R}^{n}: v^{T} p=h_{V}(p)\right\}$ is called a supporting hyperplane with exterior unit normal vector $p$. The intersection $H(p) \cap V$ is always non empty. Thus the maximized Hamiltonian can be written as $H\left(S(t), p, p^{0}, v(t)\right)=h_{V}(p)-p^{0}$ and the set $\partial V_{p}$ is equal to $H(p) \cap V$.

Let us now analyse in details the possible form of the extremal trajectories. From (8), the target point $S(t(v))$ of an extremal trajectory is always on the edge of the target. Two cases may occur: either $S(t(v))$ is on the corner of the target or it is interior to the edge. We will begin by the second case and introduce first a definition.

\section{Definition 1}

We say that the point $\bar{S}$ is under (respectively above) the cone $\mathbb{R}_{+} \partial V_{p}$ if either $\bar{S} \in \mathbb{R}_{+} \partial V_{p}$ or $\bar{S}$ lies between the cone $\mathbb{R}_{+} \partial V_{p}$ and the horizontal axis $\left(O S_{1}\right)$ (respectively the vertical axis $\left(O S_{2}\right)$ ).

\section{Lemma 2}

Let $S(\cdot)$ be a minimizing trajectory of $\left(\mathcal{P}_{c o}\right)$ with control $v(\cdot)$ such that $S(t(v)) \neq \bar{S}$.

(i) If $S(t(v)) \in\left\{S_{1}=\bar{S}_{1}\right\} \times\left\{S_{2}>\bar{S}_{2}\right\}$, then $t(v)=\frac{\bar{S}_{1}}{v_{1}^{\max }}$ and $v(t) \in \partial V_{e_{1}}$ for a.e. $t \in[0, t(v)]$. In that case, $\bar{S}$ is under the cone $\mathbb{R}_{+} \partial V_{e_{1}}$. 
(ii) If $S(t(v)) \in\left\{S_{1}>\bar{S}_{1}\right\} \times\left\{S_{2}=\bar{S}_{2}\right\}$, then $t(v)=\frac{\bar{S}_{2}}{v_{2}^{\max }}$ and $v(t) \in \partial V_{e_{2}}$ for a.e. $t \in[0, t(v)]$. In that case, $\bar{S}$ is above the cone $\mathbb{R}_{+} \partial V_{e_{2}}$.

\section{Proof}

We only give the proof for the case $(i)$, the same argument can be used to prove $(i i)$. Let us consider an extremal trajectory $S(\cdot)$ that reaches the target at a point on the open half-line $\left\{S_{1}=\bar{S}_{1}\right\} \times\left\{S_{2}>\bar{S}_{2}\right\}$ and $p$ an adjoint vector associated to $S(\cdot)$. Then, from the transversality condition and since $\|p\|=1$, we derive that $p=e_{1}=(1,0)$. This implies that $v(t) \in \partial V_{e_{1}}$ a.e. and that $v_{1}(t)=v_{1}^{\max }$. As a consequence, the trajectory $S(\cdot)$ satisfies:

$$
\dot{S}(t)=\left(v_{1}^{\max }, v_{2}(t)\right) \in \partial V_{e_{1}} .
$$

Integrating equation (10) on $[0, t(v)]$ and noticing that $S_{1}(t(v))=\bar{S}_{1}$, we obtain $S_{1}(t(v))=v_{1}^{\max } t(v)=\bar{S}_{1}$, and then $t(v)=\frac{\bar{S}_{1}}{v_{1}^{\max }}$. Moreover, $S(t(v)) \in \mathbb{R}_{+} \partial V_{e_{1}}$ which implies that $\bar{S}$ is under the cone $\mathbb{R}_{+} \partial V_{e_{1}}$.

Let $v_{\bar{S}}$ the control defined by

\section{Lemma 3}

$$
v_{\bar{S}}=\operatorname{argmax}\{\|v\|: v \in V \cap(O \bar{S})\} .
$$

Let $S(\cdot)$ be a minimizing trajectory of $\left(\mathcal{P}_{c o}\right)$ with control $v(\cdot)$ such that $S(t(v))=\bar{S}$. Then, there exists $p \in S^{1+}$ such that $\bar{S} \in \mathbb{R}_{+} \partial V_{p}$. Moreover, $t(v)=\frac{\|\bar{S}\|}{\left\|v_{\bar{S}}\right\|}$ and we have the following alternative:

- either $\partial V$ is a segment in a neighbourhood of $v_{\bar{S}}$ : in this case there is a unique vector $p \in S^{1+}$ such that $v_{\bar{S}}$ belongs to the interior of the segment $\partial V_{p}$, and $v(t) \in \partial V_{p}$ for a.e. $t \in[0, t(v)]$;

- or $v(\cdot) \equiv v_{\bar{S}}$.

Proof

The trajectory $S(\cdot)$ is an extremal trajectory, thus there exists $p \in S^{1+}$ such that $v(t) \in \partial V_{p}$ for a.e. $t \in[0, t(v)]$. This implies that $\bar{S} \in \mathbb{R}_{+} \partial V_{p}$ and $v_{\bar{S}} \in \partial V_{p}$. As a consequence $p$ belongs to the normal cone $\mathcal{N}_{V}\left(v_{\bar{S}}\right)$ to $V$ at $v_{\bar{S}}$ defined by:

$$
\mathcal{N}_{V}\left(v_{\bar{S}}\right)=\left\{p \in S^{1+}:\left\langle p, v-v_{\bar{S}}\right\rangle \leq 0, \quad \forall v \in V\right\} .
$$

Two cases must be distinguished.

- If $\partial V$ is smooth in $v_{\bar{S}}$, then $\mathcal{N}_{V}\left(v_{\bar{S}}\right)$ is a singleton, i.e. $\mathcal{N}_{V}\left(v_{\bar{S}}\right)=\left\{p^{0}\right\}$. So, we have

$$
p_{1}^{0} \bar{S}_{1}+p_{2}^{0} \bar{S}_{2}=\int_{0}^{t(v)} p^{0^{T}} v(s) d s=t(v) p^{0},
$$

and $t(v)=\frac{p_{1}^{0} \bar{S}_{1}+p_{2}^{0} \bar{S}_{2}}{p^{0}}$ since $p^{0} \neq 0$. The uniqueness of $p^{0}$ allows to conclude that $t(v)$ does not depend on the control $v$ and that all optimal trajectories reach the point $\bar{S}$ at the same time. In particular, $\bar{S}_{1}=v_{1 \bar{S}} t(v)$ and $\bar{S}_{2}=v_{2} \bar{S} t(v)$, where $v_{\bar{S}}=\left(v_{1 \bar{S}}, v_{2 \bar{S}}\right)$. This implies $t(v)=\frac{\|\bar{S}\|}{\left\|v_{\bar{S}}\right\|}$.

Two possibility occurs: either $\partial V_{p}$ is a segment containing in its interior the point $v_{\bar{S}}$, and then $v(t) \in \partial V_{p}$ for a.e. $t \in[0, t(v)]$; or $\partial V_{p}$ is the singleton $\left\{v_{\bar{S}}\right\}$ and in this last case $v(\cdot) \equiv v_{\bar{S}}$.

- If $\partial V$ is not smooth in $v_{\bar{S}}$, then there exist $\theta_{0}$ and $\theta_{1}$ in $[0, \pi / 2]$ such that the normal cone to $V$ at $v_{\bar{S}}$ is written as:

$$
\mathcal{N}_{V}\left(v_{\bar{S}}\right)=\left\{e^{i \theta}: \theta \in\left[\theta_{0}, \theta_{1}\right]\right\} .
$$

If $p \in\left\{e^{i \theta}: \theta \in\left(\theta_{0}, \theta_{1}\right)\right\}$, then $\partial V_{p}=\left\{v_{\bar{S}}\right\}$ and so $v(\cdot) \equiv v_{\bar{S}}$. The trajectory associated to such a constant control reaches the target in time $t(v)=\frac{\|\bar{S}\|}{\left\|v_{\bar{S}}\right\|}$.

In the case where $p=e^{i \theta_{0}}$, two situations may occur: either $\partial V_{e^{i \theta_{0}}}$ is equal to $\left\{v_{\bar{S}}\right\}$ and in this case $v(\cdot) \equiv v_{\bar{S}}$; or $\partial V_{e^{i \theta_{0}}}$ is a segment. In the latter case, $v_{\bar{S}}$ is an extremity of the segment and necessarily $v(\cdot)=v_{\bar{S}}$ (it is the only control with values in $\partial V_{e^{i \theta_{0}}}$ that allows to reach $\left.\bar{S}\right)$. As a consequence $t(v)=\frac{\|\bar{S}\|}{\left\|v_{\bar{S}}\right\|}$.

The case where $p=e^{i \theta_{1}}$ is treated in the same way. 


\subsection{Optimal synthesis of $\left(\mathcal{P}_{c o}\right)$}

We are now in a position to construct the optimal synthesis of the convex problem $\left(\mathcal{P}_{c o}\right)$. We distinguish three different subsets of the positive quadrant $(0,+\infty)^{2}$.

\section{Definition 2}

We define the partition $Z_{0} \cup Z_{1} \cup Z_{2}$ of $(0,+\infty)^{2}$ by setting:

- $Z_{0}$ is the union of all cones $\mathbb{R}_{+} \partial V_{p}$ for $p$ different from $e_{1}$ and $e_{2}$, that is,

$$
Z_{0}=\bigcup\left\{\mathbb{R}_{+} \partial V_{p}: p \in S^{1+}, p \neq e_{1}, e_{2}\right\} ;
$$

- $Z_{1}$ is the set of points of $(0,+\infty)^{2}$ lying under the cone $\mathbb{R}_{+} \partial V_{e_{1}}$;

- $Z_{2}$ is the set of points of $(0,+\infty)^{2}$ lying above the cone $\mathbb{R}_{+} \partial V_{e_{2}}$.

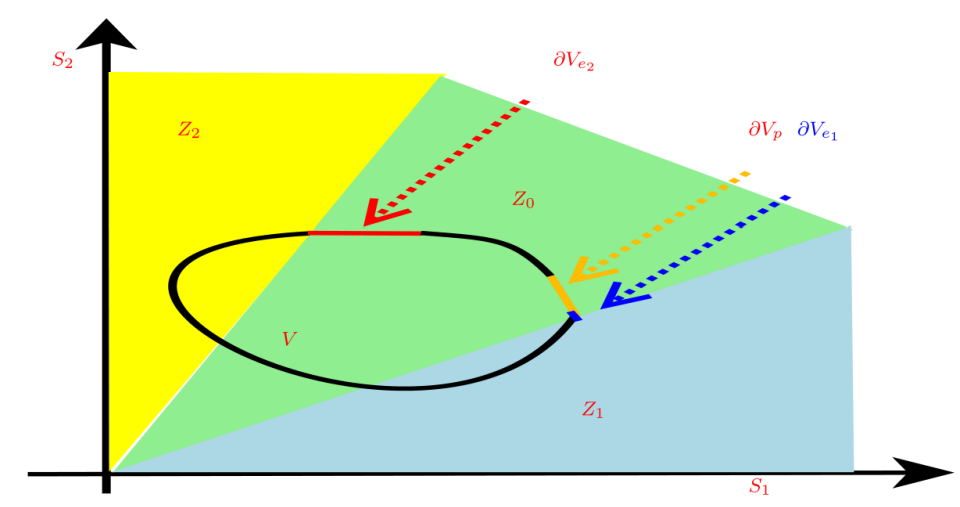

Figure 3. Partition of $\left(\mathbb{R}_{+}\right)^{2}$ into three regions $Z_{2}$ (in yellow), $Z_{0}$ (in green) et $Z_{1}$ (in blue).

\section{Theorem 1}

Let $S(\cdot)$ be a minimizing trajectory of $\left(\mathcal{P}_{c o}\right)$, and $v(\cdot)$ be the corresponding control defined on $[0, t(v)]$.

- If $\bar{S} \in Z_{0}$, then $t(v)=\frac{\|\bar{S}\|}{\left\|v_{\bar{S}}\right\|}$ and $v(t) \in \partial V_{p}$ for a.e. $t \in[0, t(v)]$, where $\partial V_{p}$ is the largest segment included in $\partial V$ and containing $v_{\bar{S}}$ in his interior; the trajectory $S(\cdot)$ reaches the target $\mathcal{C}_{\bar{S}}$ at $\bar{S}$, i.e. $S(t(v))=\bar{S}$.

- If $\bar{S} \in Z_{1}$, then $t(v)=\frac{\bar{S}_{1}}{v_{1}^{\max }}$ and $v(t) \in \partial V_{e_{1}}$ for a.e. $t \in[0, t(v)]$; the trajectory $S(\cdot)$ reaches the target $\mathcal{C}_{\bar{S}}$ on the intersection of the half-line $\left\{S_{1}=\bar{S}_{1}\right\} \times\left\{S_{2} \geq \bar{S}_{2}\right\}$ with the cone $\mathbb{R}_{+} \partial V_{e_{1}}$.

- If $\bar{S} \in Z_{2}$, then $t(v)=\frac{\bar{S}_{2}}{v_{2}^{\max }}$ and $v(t) \in \partial V_{e_{2}}$ for a.e. $t \in[0, t(v)]$; the trajectory $S(\cdot)$ reaches the target $\mathcal{C}_{\bar{S}}$ on the intersection of the half-line $\left\{S_{1} \geq \bar{S}_{1}\right\} \times\left\{S_{2}=\bar{S}_{2}\right\}$ with the cone $\mathbb{R}_{+} \partial V_{e_{2}}$.

\section{Remark 2}

If $\partial V$ contains no segment then the optimal control is constant, and its value only depends on the position of the point $\bar{S}$ with respect to the set $V$. It can be determined geometrically, see Figure 4 .

\section{Proof}

If $\bar{S} \in Z_{0}$, then $\bar{S}$ is neither under $\mathbb{R}_{+} \partial V_{e_{1}}$ nor above $\mathbb{R}_{+} \partial V_{e_{2}}$, which implies that $S(\cdot)$ satisfy the hypothesis of Lemma 2. As a consequence, Lemma 3 applies and we get the conclusion.

Let us consider the case $\bar{S} \in Z_{1}$ (the case $\bar{S} \in Z_{2}$ can be treated in the same manner). If $\bar{S} \notin \mathbb{R}_{+} \partial V_{e_{1}}$, then only the conclusion of Lemma 2-(i) applies and the result follows. Assume now that $\bar{S} \in \mathbb{R}_{+} \partial V_{e_{1}}$. Two situations 

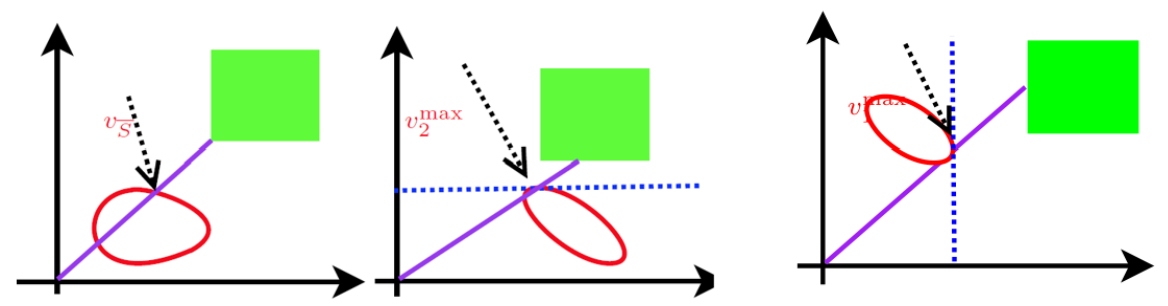

Figure 4. Possible cases for a constant control.

may occur: either $S(t(v))=\bar{S}$, and then from Lemma 3 we deduce that $v(t) \in \partial V_{e_{1}}$ a.e. and that $t(v)=\frac{\|\bar{S}\|}{\left\|v_{\bar{S}}\right\|}$; or $S(t(v)) \in\left\{S_{1}=\bar{S}_{1}\right\} \times\left\{S_{2}>\bar{S}_{2}\right\}$, and it results from Lemma $2-(i)$ that $v(t) \in \partial V_{e_{1}}$ a.e. and that $t(v)=\frac{\bar{S}_{1}}{v_{1}^{\max }}$. Since $\frac{\|\bar{S}\|}{\left\|v_{\bar{S}}\right\|}=\frac{\bar{S}_{1}}{v_{1}^{\max }}$, both cases give the same conclusion., and the proof is completed.

\subsection{Back to problem $(\mathcal{P})$}

We come back now to problem $(\mathcal{P})$. We choose $V$ to be the convex hull of the set $\left\{F(u): u \in\left[0, u_{\max }\right]\right\}$ and we denote by $\left(\mathcal{P}_{c o}\right)$ the associated minimum time problem. Thus we are considering two minimum time problems with the same initial condition $S(0)=0$ and the same $\operatorname{target} \mathcal{C}_{\bar{S}}$ but the control system in $(\mathcal{P})$ is

$$
\dot{S}=v, \quad v \in\left\{F(u): u \in\left[0, u_{\max }\right]\right\},
$$

whereas the control system in $\left(\mathcal{P}_{c o}\right)$ is

$$
\dot{S}=v, \quad v \in V=\operatorname{conv}\left\{F(u): u \in\left[0, u_{\max }\right]\right\} .
$$

Proposition 1

Let $t_{\text {min }}\left(\mathcal{P}_{c o}\right)$ and $t_{\text {min }}(\mathcal{P})$ be the minimal times corresponding to problems $\left(\mathcal{P}_{c o}\right)$ and $(\mathcal{P})$, respectively. Then,

$$
t_{\text {min }}\left(\mathcal{P}_{c o}\right)=t_{\min }(\mathcal{P}) \text {. }
$$

Proof

Since the set of admissible velocities for $\left(\mathcal{P}_{c o}\right)$ contains the one of $(\mathcal{P})$, we have

$$
t_{\text {min }}\left(\mathcal{P}_{\text {co }}\right) \leq t_{\text {min }}(\mathcal{P})
$$

so we have to prove the converse inequality. From Theorem $1,\left(\mathcal{P}_{c o}\right)$ admits at least one optimal solution $S^{*}(\cdot)$ associated with a constant control $v^{*} \in V\left(v^{*}=v_{\bar{S}}\right.$ if $\bar{S} \in Z_{0}, v^{*} \in \partial V_{e_{1}}$ if $\bar{S} \in Z_{1}$, and $v^{*} \in \partial V_{e_{2}}$ if $\left.\bar{S} \in Z_{2}\right)$. Thus the trajectory is $S^{*}(t)=t v^{*}, t \in\left[0, t^{*}\right]$ with $t^{*}=t_{\min }\left(\mathcal{P}_{c o}\right)$. By definition of $V$, we have $v^{*}=$ $\lambda F\left(u_{1}\right)+(1-\lambda) F\left(u_{2}\right)$, where $\lambda \in[0,1]$ and $u_{1}, u_{2} \in\left[0, u_{\max }\right]$. Note that $u_{1}=u_{2}$ when $\partial V$ is not a segment near $v^{*}$. Let us define the function $u:\left[0, t^{*}\right] \rightarrow\left[0, u_{\max }\right]$ by:

$$
\begin{cases}u(t)=u_{1} & \text { for } \quad t \in\left[0, \lambda t^{*}\right] \\ u(t)=u_{2} & \text { for } \quad t \in\left[\lambda t^{*}, t^{*}\right] .\end{cases}
$$

The solution $S(\cdot)$ of $\dot{S}(t)=F(u(t)), S(0)=0$, is an admissible trajectory for the problem $\mathcal{P}$ and satisfies $S\left(t^{*}\right)=S^{*}\left(t^{*}\right) \in \mathcal{C}_{\bar{S}}$. We deduce that $t_{\text {min }}(\mathcal{P}) \leq t^{*}=t_{\text {min }}\left(\mathcal{P}_{c o}\right)$, which concludes the proof.

This result allows to solve the original problem $\left(\mathcal{P}_{0}\right)$. Indeed, let us define the homeomorphism $\varphi$ by:

$$
\begin{aligned}
\varphi:(0,+\infty)^{2} & \rightarrow(0,+\infty)^{2} \\
\left(s_{1}, s_{2}\right) & \mapsto\left(\int_{a_{1}}^{s_{1}} \frac{d s}{\rho_{1}(s)}, \int_{a_{2}}^{s_{2}} \frac{d s}{\rho_{2}(s)}\right) .
\end{aligned}
$$


Then the optimal value of the problem $\left(\mathcal{P}_{0}\left(s^{0}\right)\right)$ obtained by starting from an initial condition $s^{0}$ is equal to the minimum time of the problem $\left(\mathcal{P}_{c o}\left(\varphi\left(s^{0}\right)\right)\right)$ obtained for the target $\mathcal{C}_{\bar{S}}$ with $\bar{S}=\varphi\left(s^{0}\right)$.

As for the minimizing controls, note that $u(\cdot)$ is minimizing for $\left(\mathcal{P}\left(\varphi\left(s^{0}\right)\right)\right.$ ) (and then for $\left(\mathcal{P}_{0}\left(s^{0}\right)\right)$ ) if and only if $v(t)=F(u(t))$ is minimizing for $\left(\mathcal{P}_{c o}\left(\varphi\left(s^{0}\right)\right)\right)$. We then obtained the corresponding optimal control of $\left(\mathcal{P}_{0}\left(s^{0}\right)\right)$ through the time-reparameterization (2). In particular, the construction (13) implies the following property.

Corollary 1

For all $s^{0} \in \mathbb{R}_{+}^{2}$, there exists an optimal piecewise constant control for problem $\left(\mathcal{P}_{0}\right)$ with at most one discontinuity.

\section{Application}

With respect to the model proposed by Mazouni, [7], we assume here that nitrification and denitrification are well controlled by oxygen and that the constraint on the organic matter needed for denitrification is ignored. This simplification allows us to handle a differential system in two dimensions and to consider an optimal control problem in minimal time and energy.

\subsection{The model}

We consider a model describing the following biological reaction scheme:

$$
\begin{aligned}
& s_{1}+u \mapsto x_{1} \\
& s_{2}+u \mapsto x_{2}
\end{aligned}
$$

where $s_{1}$ and $s_{2}$ are the concentrations of the substrates to degrade, $x_{1}$ and $x_{2}$ are those of the bacteria consuming $s_{1}$ and $s_{2}$, respectively, for their growth. $u$ is the oxygen concentration. The first reaction is enhanced by the presence of oxygen whereas the second is inhibited by the oxygen.

Assuming that the system is operating in batch mode, the mathematical model is given by:

$$
\left\{\begin{array}{c}
\dot{x}_{1}=\nu_{1}\left(s_{1}, u\right) x_{1}, \\
\dot{s}_{1}=-\nu_{1}\left(s_{1}, u\right) x_{1}, \\
\dot{x}_{2}=\nu_{2}\left(s_{2}, u\right) x_{2}, \\
\dot{s}_{2}=-\nu_{2}\left(s_{2}, u\right) x_{2},
\end{array}\right.
$$

where $\nu_{1}$ and $\nu_{2}$ are the specific growth function of $x_{1}$ and $x_{2}$, respectively. We assume that:

$\mathbf{H}_{\mathbf{1}}: \nu_{1}\left(s_{1}, u\right)=\mu_{1}\left(s_{1}\right) f_{1}(u)$ and $\nu_{2}\left(s_{2}, u\right)=\mu_{2}\left(s_{2}\right) f_{2}(u)$, with $f_{1}$ and $f_{2}$ satisfying Hypothesis $\mathbf{H}$ and $\mu_{1}$ and $\mu_{2}$ are positive, continuous with $\mu_{1}(0)=\mu_{2}(0)=0$.

$\mathbf{H}_{2}: 0 \leq u(t) \leq 1$

$\mathbf{H}_{3}:$ For all $u \in[0,1]$ and $s_{1} \geq 0, \frac{\partial \nu_{1}}{\partial u}\left(s_{1}, u\right)>0, \nu_{1}\left(s_{1}, 0\right)=0$.

$\mathbf{H}_{4}:$ For all $u \in[0,1]$ and $s_{2} \geq 0, \frac{\partial \nu_{2}}{\partial u}\left(s_{2}, u\right)<0$.

Hypothesis $\mathbf{H}_{1}$ together with $\mathbf{H}_{3}$ and $\mathbf{H}_{4}$ imply that $f_{1}^{\prime}(u)>0$ and $f_{2}^{\prime}(u)<0$, for all $u \in[0,1]$. For a sake of simplicity, we assumed in $\mathbf{H}_{2}$ that the maximal oxygen concentration injected in the bio-reactor $u_{\max }=1$. Hypothesis $\mathbf{H}_{3}$ means that $\nu_{1}$ increases continuously when the oxygen quantity injected into the reactor also increases and that there is no variation of $s_{1}$ without oxygen. $\mathbf{H}_{4}$ means that $\nu_{2}$ decreases continuously when the oxygen quantity increases.

Hypothesis $\mathbf{H}_{\mathbf{1}}, \mathbf{H}_{\mathbf{3}}$ and $\mathbf{H}_{\mathbf{4}}$ are fulfilled, for example, by growth rates $\mu_{i}$ of Monod-type $\mu_{\max } \frac{s_{i}}{K_{s}+s_{i}}$ or of Haldane-type $\mu_{\max } \frac{s_{i}}{K_{s}+s_{i}+K_{i} / s_{i}^{2}}, i=1,2$ and by functions $f_{1}$ of Monod-type $f_{\max } \frac{u}{K_{s}+u}$ and $f_{2}$ of the 
form $f_{\max } \frac{K_{s}}{K_{s}+u}$. Note that, by mass conservation in system (16), we have: $\dot{s}_{i}(t)+\dot{x}_{i}(t)=0$, for $i=1,2$ and $t \geq 0$. We set then $M_{i}=s_{i}(0)+x_{i}(0)$, for $i=1,2$. Without loss of generality, we assume that $M_{i}=1$, for $i=1,2$. System (16) is then reduced to the bidimensional system:

$$
\left\{\begin{array}{l}
\dot{s}_{1}=-f_{1}(u) \rho_{1}\left(s_{1}\right), \\
\dot{s}_{2}=-f_{2}(u) \rho_{2}\left(s_{2}\right),
\end{array}\right.
$$

where $\rho_{1}\left(s_{1}\right)=\mu_{1}\left(s_{1}\right)\left(1-s_{1}\right)$ and $\rho_{2}\left(s_{2}\right)=\mu_{2}\left(s_{2}\right)\left(1-s_{2}\right)$. This implies in particular that $\rho_{i}(0)=\rho_{i}(1)=0$, $i=1,2$. System (17) has the same form as system (1) considered in Section 2.

Our objective is as follows: given normative constraints $\bar{s}_{i}>0$, from any initial condition $x_{i}(0)>0, s_{i}(0)>\bar{s}_{i}$, one seeks to synthesize the control $u(t)$ such that $s_{i}$ be smaller or equal to $\bar{s}_{i}>0$ in minimizing a function of both time and energy.

The functions $\mu_{1}$ and $\mu_{2}$ are the degradation rates of the substrates which concentrations must be controlled. The choice of growth functions essentially depends on the nature of the treated substrates. In the context of wastewater treatment, the limitation of the nitrogen oxidation by oxygen (or nitrification) is generally modeled by a Monod-type function $\mu_{\max } \frac{\mathrm{O}_{2}}{\mathrm{~K}_{s}+\mathrm{O}_{2}}$ multiplied by the growth function. Similarly, the substrate inhibition by oxygen is described by the $\mu_{\max } \frac{K_{s}}{K_{s}+O_{2}}$. Thus, the limitation of the substrate degradation is modeled by the product of the last function in oxygen and a Monod-type function in nitrogen.

Remark 3

If a substrate $s$ is inhibiting the process at high concentrations, the inhibition can be modeled by the Haldane-type growth function $\mu_{\max } \frac{s}{K_{s}+s+K_{I} / s^{2}}$.

\subsection{The optimal control problem}

We consider cost functions of the form:

$$
C(u)=\int_{0}^{t(u)}\left(1+\alpha u^{2}(s)\right) d s=t(u)+\alpha \int_{0}^{t(u)} u^{2}(s) d s,
$$

where $\alpha$ is a given nonnegative parameter. We take $J(u)=J_{\alpha}(u)=\alpha u^{2}$, in problem $\mathcal{P}_{0}$ of Section 2 . This cost function expresses a compromise between the time to reach the target and the oxygen energy consumed during this duration.

For $\alpha \geq 0$, we define then the following optimal control problem:

$$
\left(\mathcal{P}^{\alpha}\right)\left\{\begin{array}{l}
\min C(u)=t(u)+\alpha \int_{0}^{t(u)} u^{2}(\tau) d \tau \\
u \in L^{\infty}([0, t(u)],[0,1]), \\
s(\cdot) \text { solution of }(17) \text { s.t. } s(0)=s^{0} \text { and } s(t(u)) \in \mathcal{T} .
\end{array}\right.
$$

$\mathcal{T}$ is the target defined by: $\mathcal{T}=\left[0, a_{1}\right] \times\left[0, a_{2}\right]$, where $a_{1}$ and $a_{2}$ represent the threshold allowed to substrate concentrations in the rejected water. With the previous choices of $f_{1}, f_{2}$ and $J$, all the curves in the set

$$
\left\{\left(\frac{f_{1}(u)}{1+J_{\alpha}(u)}, \frac{f_{2}(u)}{1+J_{\alpha}(u)}\right) ; \quad u \in[0,1]\right\},
$$

are strictly convex curves. Moreover, from Remark 2, the optimal control corresponding to problem $\left(\mathcal{P}^{\alpha}\right)$ is constant. The region $Z_{2}^{\alpha}$ is empty, and $Z_{0}^{\alpha}$ and $Z_{1}^{\alpha}$ form a partition of $\mathbb{R}_{+}^{2}$. Then, the optimal control value depends 
on the region $\bar{S}$ belongs to.

The initial concentrations $s_{1}^{0}=s_{1}(0)$ et $s_{2}^{0}=s_{2}(0)$ are both above the threshold which defines the target $\mathcal{T}$, see Section 2. This means that $s_{1}^{0} \geq a_{1}$ and $s_{2}^{0} \geq a_{2}$. That's why, we will restrict our study only to the subspace $D$ defined by:

$$
D=\left\{s^{0} \in \mathbb{R}_{+}^{2}, s_{1}^{0} \geq a_{1} \text { and } s_{2}^{0} \geq a_{2}\right\} .
$$

Therefore, we consider the restriction of the homeomorphism $\varphi$ on $D$ defined by:

$$
\begin{aligned}
\varphi: D & \rightarrow(0,+\infty)^{2} \\
\left(s_{1}, s_{2}\right) & \mapsto\left(\int_{a_{1}}^{s_{1}} \frac{d s}{\rho_{1}(s)}, \int_{a_{2}}^{s_{2}} \frac{d s}{\rho_{2}(s)}\right) .
\end{aligned}
$$

We set $\mathcal{Z}_{i}^{\alpha}=\varphi^{-1}\left(Z_{i}^{\alpha}\right)$, for $i \in\{0,1\}$. These regions define a partition of $D$. For all $s^{0} \in \mathcal{Z}_{1}^{\alpha}$, the minimizing trajectory $s_{\bar{u}}(\cdot)$ starting from $s^{0}$ and corresponding to the control:

$$
\bar{u}=\underset{u \in[0,1]}{\operatorname{argmax}} \frac{f_{1}(u)}{1+J_{\alpha}(u)}
$$

reaches the target $\mathcal{T}$ at a point in $\left\{s_{1}=a_{1}\right\} \times\left\{s_{2}<a_{2}\right\}$. If $s^{0} \notin \mathcal{Z}_{1}^{\alpha}$, the minimizing trajectories starting from $s^{0}$ reach the target at the corner $\left(a_{1}, a_{2}\right)$.

\subsection{Numerical results}

In the following, the function $f_{1}$ is of Monod-type, the function $f_{2}$ expresses the effect of oxygen inhibition at high concentrations in oxygen. They are defined on $[0,1]$ by:

$$
f_{1}(u)=\frac{u}{0.2+u} \quad \text { and } \quad f_{2}(u)=\frac{0.1}{0.1+u} .
$$

The target $\mathcal{T}$ is given by $\mathcal{T}=[0,0.1] \times[0,0.1]$, the initial condition is $\left(s_{1}^{0}, s_{2}^{0}\right)=(0.65,0.5)$. In the following, we consider the cases $\alpha=0$ and $\alpha=20$, first when $\mu_{1}$ et $\mu_{2}$ are of Monod-type and then when $\mu_{1}$ is of Haldane-type and $\mu_{2}$ is of Monod-type.

4.3.1. Case where $\mu_{1}$ and $\mu_{2}$ are of Monod-type In this part, we assume that the growth functions $\mu_{1}$ and $\mu_{2}$ are defined on $[0,1]$ and are of Monod-type:

$$
\mu_{1}(x)=0.5 \frac{x}{1+x} \quad \text { and } \quad \mu_{2}(x)=0.8 \frac{x}{1+x} .
$$

We present in Figure 5 and Figure 6 the regions $Z_{0}^{\alpha}$ and $Z_{1}^{\alpha}$ with the corresponding regions $\mathcal{Z}_{0}^{\alpha}$ and $\mathcal{Z}_{1}^{\alpha}$, for $\alpha=0$ and $\alpha=20$, respectively.

In the case $\alpha=0$, the initial condition $s^{0}$ is in $\mathcal{Z}_{0}^{0}$, the minimizing trajectory reaches the target at the point $\left(a_{1}, a_{2}\right)$. But, when $\alpha=20, s^{0} \in Z_{1}^{20}$ the minimizing trajectory reaches the target on the edge $\left.\left\{s_{1}=0.1\right\} \times\right] 0,0.1[$.

If $\alpha=20$, the minimizing trajectory takes more time to reach the target than in the case of minimal time trajectories $(\alpha=0)$, as shown in Figure 7. In this case, since we take into account the energy, the control value is lower than in the case $\alpha=0$. The growth of $x_{2}$ is thus less inhibited than in the case $\alpha=0$. The rate at which $s_{2}$ decreases is then faster than the rate at which $s_{1}$ decreases.

Interestingly, we note the existence of a critical value $\alpha^{*}$ such that $t^{\alpha}$ is constant up to $\alpha^{*}$ and increases for $\alpha \geq \alpha^{*}$. Indeed, for $\alpha \leq \alpha^{*}, s^{0}$ is in the region $D \backslash \mathcal{Z}_{1}^{\alpha}$. Then, the minimal time to reach the target is given by 

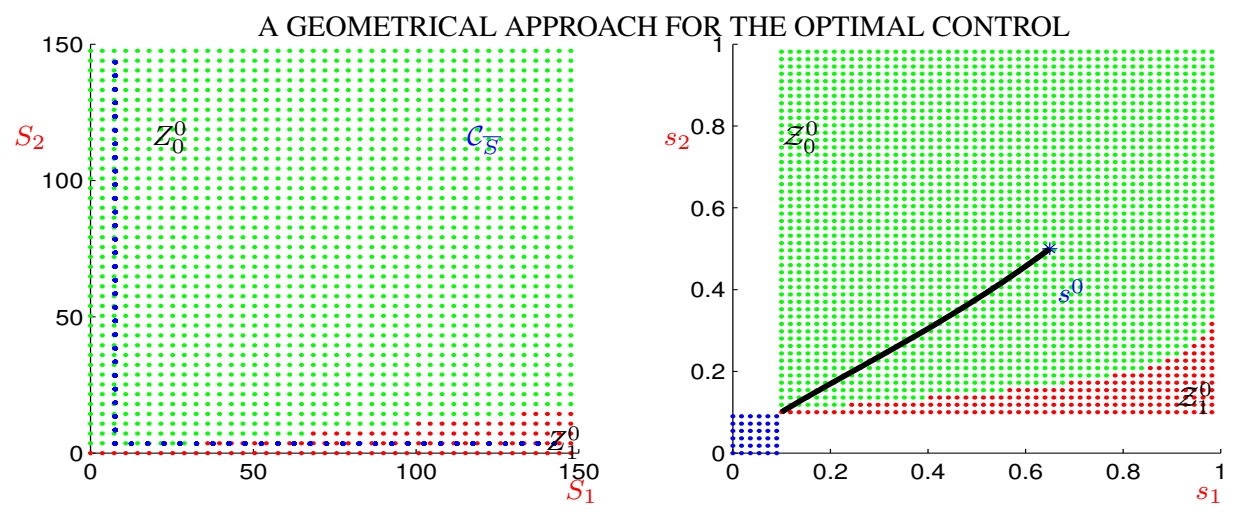

Figure 5. The regions $Z_{0}^{0}, Z_{1}^{0}, \mathcal{Z}_{1}^{0}$ and $\mathcal{Z}_{0}^{0}$.
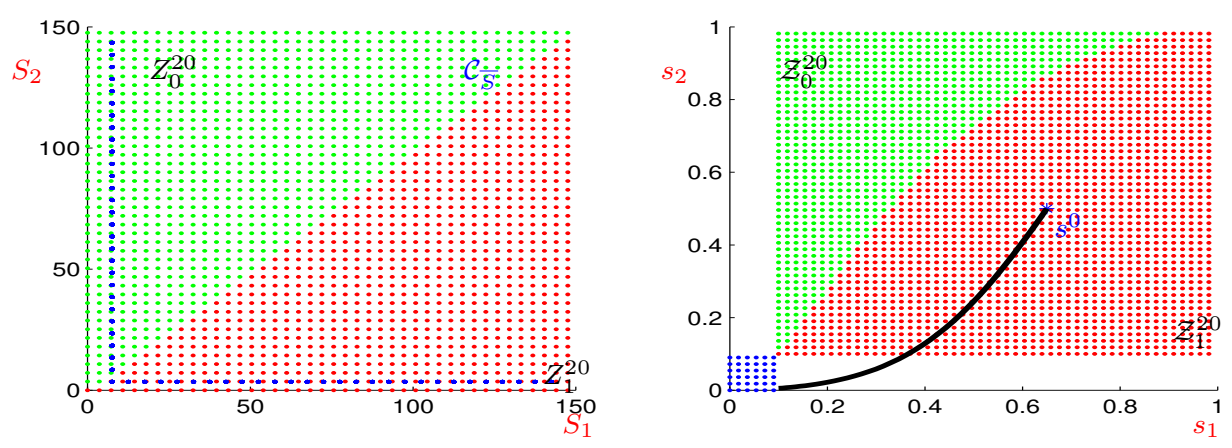

Figure 6. The regions $Z_{0}^{20}, Z_{1}^{20}, \mathcal{Z}_{1}^{20}$ and $\mathcal{Z}_{0}^{20}$.

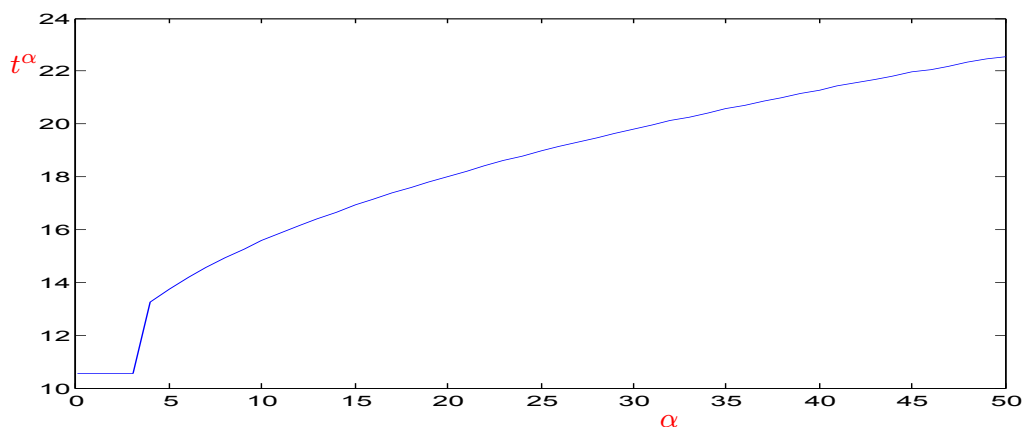

Figure 7. The time $t^{\alpha}$ required by the minimizing trajectory to reach the target as a function of $\alpha$.

$t_{\text {min }}=\frac{\|\bar{S}\|}{v_{\bar{S}}}$, where $v_{\bar{S}}=\underset{v \in V \cap(O \bar{S})}{\operatorname{argmax}}\|v\|$. This value does not depend on $\alpha$. Now, if $\alpha>\alpha^{*}$ then $s^{0} \in \mathcal{Z}_{1}^{\alpha}$ and the time taken for the minimizing trajectory to reach the target is given by $t_{\min }=\frac{\left\|\bar{S}_{1}\right\|}{f_{1}(\bar{u})}$ where $\bar{u}=\underset{u \in[0,1]}{\operatorname{argmax}}\left\{\frac{f_{1}(u)}{1+J_{\alpha}(u)}\right\}$.

4.3.2. Case where $\mu_{1}$ is of Haldane-type and $\mu_{2}$ is of Monod-type Figures 8 and 9 are obtained with a growth function $\mu_{1}$ corresponding to the inhibition of the substrate (function Haldane-type) and a growth function $\mu_{2}$ of Monod-type. 

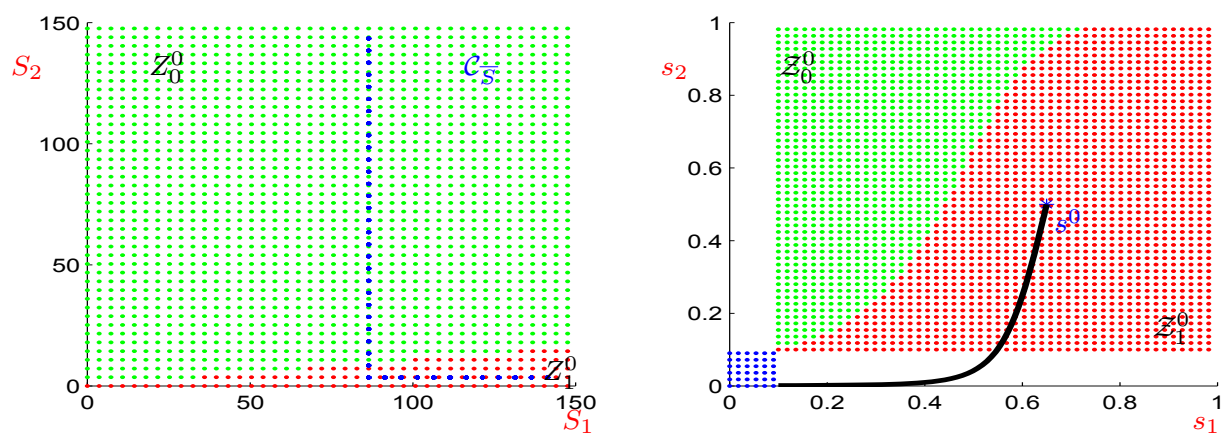

Figure 8. The regions $Z_{0}^{0}, Z_{1}^{0}, \mathcal{Z}_{0}^{0}$ and $\mathcal{Z}_{1}^{0}$.

In this case, the region $Z_{1}^{0}$ (respectively $\mathcal{Z}_{1}^{0}$ ) is more important than the region $Z_{0}^{0}$ (respectively $\mathcal{Z}_{0}^{0}$ ). Moreover, for $\alpha=0, s^{0} \in \mathcal{Z}_{1}^{0}$. Since $\mathcal{Z}_{1}^{0} \subset \mathcal{Z}_{1}^{\alpha}$, for all $\alpha \geq 0$. Then, for all $\alpha>0$, the minimizing trajectory reaches the target on the edge $\left.\left\{s_{1}=0.1\right\} \times\right] 0,0.1[$ and the minimal time depends on $\alpha$.
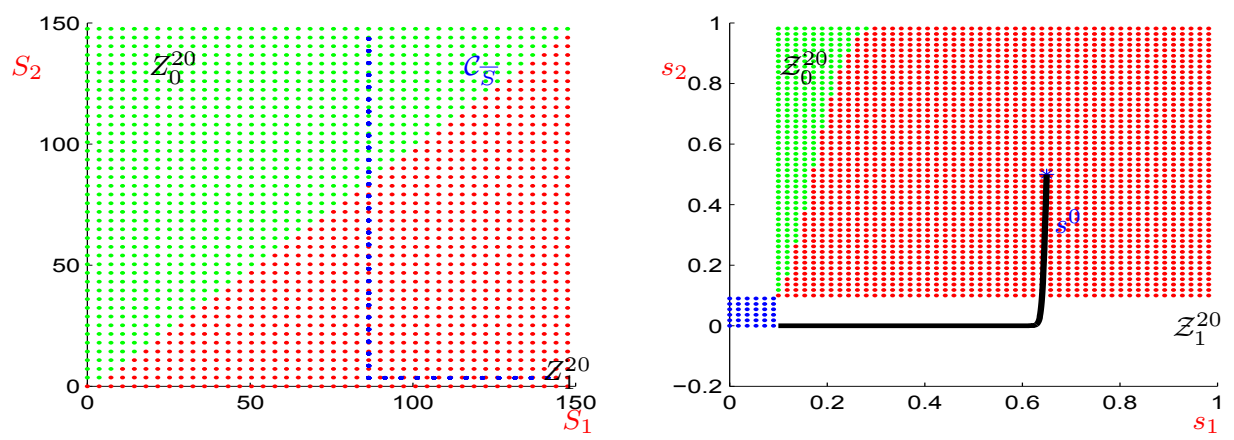

Figure 9. The regions $Z_{0}^{20}, Z_{1}^{20}, \mathcal{Z}_{1}^{20}$ and $\mathcal{Z}_{0}^{20}$.

In this case, the shapes of the trajectory discussed in the previous section are amplified in the sense the decrease of $s_{2}$ is still faster. Indeed, $s_{1}$ inhibits the growth of $x_{1}$ which diminish its decrease rate. Putting the inhibition on $x_{2}$ would lead to the opposite tendency.

From the different cases we considered, we noticed that the regions $\mathcal{Z}_{1}^{\alpha}$ increase when $\alpha$ increases. When working with two species of different nature, a first one with an inhibitory growth function in substrate and a limited one in oxygen, and the second species with limited growth functions in substrate and oxygen, the reduction of oxygen consumption leads to an increase in the duration of the reaction, for a large set of initial conditions. In fact, for $s^{0} \in \mathcal{Z}_{1}^{\alpha}$, the necessary time to reach the target depends on $\alpha$ and is no longer minimal.

More general comments are difficult to establish since the optimal trajectories depend not only on the objective function (and thus on $\alpha$ ) but also on model structure parameters. Finally, the most important result is that in any case, the optimal control is constant.

\section{Conclusion}

In this paper, we propose a model that involves two biological reactions, one being aerobic and the other anoxic, required for processing two types of substrates. The control is the dissolved oxygen considered as an input taking continuous values. The objective is to minimize a compromise between the total reaction time and the energy consumption. The dynamics are described by a differential system coupled in the control variable and decoupled in the state variables. Our approach consists in studying first a more general optimal control problem with a cost function being the integral of a positive function $J$, using a geometric technique. Due to the maximum principle, we have characterized the extremal trajectories and we determined the minimal time to reach the target and the corresponding control, according to the position of the target point on the edge of the target. This technique 
allows us to solve the problem in a very general framework (only the assumption of continuity of the growth functions in oxygen is required). Using these results, we determined the optimal strategy to control the nitrificationdenitrification reactions by oxygen, under the assumption that the organic matter is not needed for denitrification. The mathematical study is followed by numerical simulations which illustrate the obtained results. The results of this first study will help us tackle the problem in three dimensions where the variables are coupled by an additional substrate (carbon).

\section{Acknowledgment}

The authors wish to thank the financial support of TREASURE euro-Mediterranean research network (http: //www.inra.fr/treasure) and of PHC UTIQUE project 13G1120.

\section{REFERENCES}

[1] . M. Aliane, N. Moussouni, M. Bentobache, Optimal control of a rectilinear motion of a rocket. Stat., Optim. Inf. Comput., Vol.8, pp 281-295, (2020).

[2] . M. BARDI, I. CAPUZZO-DolcetTA. Optimal Control and Visosity Solutions of Hamilton-Jacobi- Bellman Equations, Birkhauser, (1997).

[3]. W. Bouhafs, N. Abdellatif, F. Jean, J. Harmand. Commande optimale en temps minimal d'un procédé biologique d'épuration de l'eau. Arima Journal, 18, pp 37-51, (2014).

[4]. W. Bouhafs, N. Abdellatif, F. Jean, J. Harmand. Commande optimale en temps et en énergie d'un procédé biologique d'épuration de l'eau. International Joint Conference CB-WR-MED Conference 2nd AOP, Tunisia Conference for Sustainable Water Management, Tunis: April, 24-27, (2013).

[5] . M. Henze, W. Gujer, T. Mino, M. van Loosdrecht. Activated sludge models ASM1, ASM2, ASM2d and ASM3, Edited by IWA Task group on mathematical modelling for design and operation of biological wastewater treatment, IWA Publishing, (2000).

[6] . O. Kostylenko, H. S. Rodrigues, D. F. M. Torres. The Risk of Contagion Spreading and its Optimal Control in the Economy. Stat., Optim. Inf. Comput., Vol.7, pp 578-587, (2019).

[7] . D. MAZouni, Thèse de doctorat - Modélisation et commande en temps minimum des réacteurs biologiques séquentiels discontinus, Université Claude Bernard-Lyon I, (2006).

[8] . D. Mazouni, J. Harmand, A. Rapaport, H. Hammouri. Optimal time control of switching systems : application to biological Sequencing Batch Reactors, Optimal Control Application and Methods, Vol. 31, No.4, pp 289-301, (2010).

[9] . J. Moreno, Optimal time control of bioreactors for the wastewater treatment. Optim. Control Appl. Meth., No 20, pp 145-164, (1999).

[10]. L. Pontryagin, V. Boltyanskit, R. Gamkrelidze, E. Mischenko, The Mathematical Theory of Optimal Processes. Wiley Interscience (1962), pp 159-160, Edition de l'école polytechnique, (2006).

[11]. R. VINTER, Optimal Control. Modern Birkhäuser Classics (2000). 